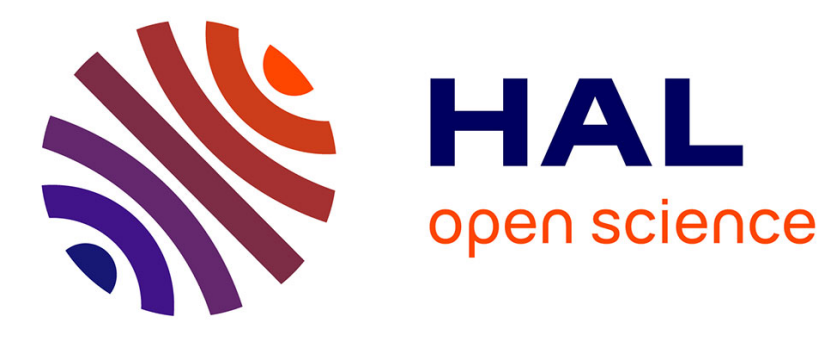

\title{
A theoretical study of linear beryllium chains: full configuration interaction.
}

\author{
Valentina Vetere, Antonio Monari, Anthony Scemama, Gian Luigi Bendazzoli, \\ Stefano Evangelisti
}

\section{- To cite this version:}

Valentina Vetere, Antonio Monari, Anthony Scemama, Gian Luigi Bendazzoli, Stefano Evangelisti. A theoretical study of linear beryllium chains: full configuration interaction.. Journal of Chemical Physics, 2009, 130 (2), pp.024301. 10.1063/1.3054709 . hal-00875603

\section{HAL Id: hal-00875603 https://hal.science/hal-00875603}

Submitted on 28 Jan 2020

HAL is a multi-disciplinary open access archive for the deposit and dissemination of scientific research documents, whether they are published or not. The documents may come from teaching and research institutions in France or abroad, or from public or private research centers.
L'archive ouverte pluridisciplinaire HAL, est destinée au dépôt et à la diffusion de documents scientifiques de niveau recherche, publiés ou non, émanant des établissements d'enseignement et de recherche français ou étrangers, des laboratoires publics ou privés. 


\section{A theoretical study of linear beryllium chains: Full configuration interaction}

Cite as: J. Chem. Phys. 130, 024301 (2009); https://doi.org/10.1063/1.3054709

Submitted: 21 October 2008 . Accepted: 02 December 2008 . Published Online: 08 January 2009

Valentina Vetere, Antonio Monari, Anthony Scemama, Gian Luigi Bendazzoli, and Stefano Evangelisti

\section{ARTICLES YOU MAY BE INTERESTED IN}

Incremental full configuration interaction

The Journal of Chemical Physics 146, 104102 (2017); https://doi.org/10.1063/1.4977727

A theoretical study of $\mathrm{Be}_{N}$ linear chains: Variational and perturbative approaches

The Journal of Chemical Physics 131, 034309 (2009); https://doi.org/10.1063/1.3185351

Gaussian basis sets for use in correlated molecular calculations. I. The atoms boron through neon and hydrogen

The Journal of Chemical Physics 90, 1007 (1989); https://doi.org/10.1063/1.456153

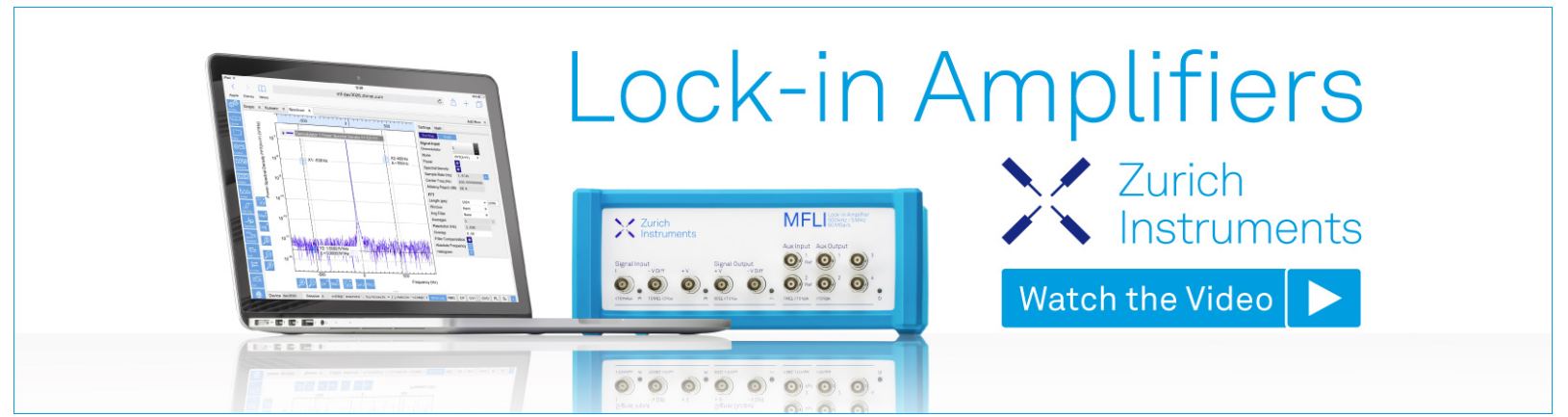

J. Chem. Phys. 130, 024301 (2009); https://doi.org/10.1063/1.3054709 


\title{
A theoretical study of linear beryllium chains: Full configuration interaction
}

\author{
Valentina Vetere, ${ }^{1}$ Antonio Monari, ${ }^{2, a)}$ Anthony Scemama, ${ }^{1}$ Gian Luigi Bendazzoli, ${ }^{2}$ and \\ Stefano Evangelisti ${ }^{1, b)}$ \\ ${ }^{1}$ Laboratoire de Chimie et Physique Quantiques-UMR 5626, Université de Toulouse et CNRS, \\ 118 Route de Narbonne, F-31062 Toulouse Cedex, France \\ ${ }^{2}$ Dipartimento di Chimica Fisica e Inorganica, Università di Bologna, Viale Risorgimento 4, \\ I-40136 Bologna, Italy
}

(Received 21 October 2008; accepted 2 December 2008; published online 8 January 2009)

\begin{abstract}
We present a full configuration interaction study of $\mathrm{Be}_{N}(N=2,3,4,5)$ linear chains. A comparative study of the basis-set effect on the reproduction of the energy profile has been reported. In particular, the $3 s 1 p, 4 s 2 p, 4 s 2 p 1 d, 5 s 3 p 2 d$, and $5 s 3 p 2 d 1 f$ bases were selected. For the smallest chains (i.e., $\mathrm{Be}_{2}$ and $\mathrm{Be}_{3}$ ), smaller basis sets give dissociative energy profiles, so large basis set is demanded for the reproduction of equilibrium minima in the structures. $\mathrm{For}_{4}$ and $\mathrm{Be}_{5}$ linear chains, the energy profiles show a minimum also by using the smallest basis sets, but the largest ones give a much stronger stabilization energy. For all the structures, two spin states have been studied: the singlet and the triplet. It is shown that the energy separation of the two states, in the equilibrium region, is small and decays exponentially with respect to the number of atoms in the chain. Finally an interpolative technique allowing for the estimation of the long-chain parameters from shorter ones is presented. (0) 2009 American Institute of Physics. [DOI: 10.1063/1.3054709]
\end{abstract}

\section{INTRODUCTION}

The interest in linear atomic structures has grown enormously in the past years since it became possible to synthesize one-dimensional electron systems at stepped surfaces. ${ }^{1-5}$ A very common feature of these systems is the presence of edge or end orbitals at the two extremities. They correspond, in a one-electron formalism, to the "surface states" introduced by Tamm and Shockley long ago. ${ }^{6}$ It should be stressed that the expression surface states are to be understood in the context of a one-electron approximation: strictly speaking, any electronic state of an extended system involves all the system electrons. Edge effects, for instance, have been predicted in graphene nanoribbons, ${ }^{7,8}$ and this can produce a large variety of interesting electric and magnetic behaviors. ${ }^{9}$ In one-dimensional systems, these states have been detected, for instance, in gold atomic chains deposited on silicon surfaces. ${ }^{5}$ For a discussion of the phenomenon of end orbitals in the case of a one-electron Hückel Hamiltonian, see Ref. 10. We present in this article a full configuration interaction (FCI) study concerning the peculiar electronic structure of linear beryllium chains $\mathrm{Be}_{N}$. In particular, in the present study, we considered values of $N$ ranging from 2 to 5 . Beryllium was chosen because of its small number of electrons (4), a fact that permits a high-level quantum description of chains containing several atoms. Preliminary calculations on elements belonging to groups 2 and 12 , however, indicate that this behavior could be a rather general one, shared by other metals of these groups.

Beryllium is a metallic divalent species. In the isolated atom, its electronic configuration is $1 s^{2} 2 s^{2}$. This is a closedshell structure, somewhat reminiscent of a rare gas, and for

\footnotetext{
a)Electronic mail: amonari@fci.unibo.it.

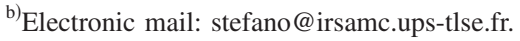

this reason this species is not very reactive. In particular, beryllium has a relatively large ionization potential, and the isolated atom does not support any stable anionic state. However, the empty $2 p$ orbitals are close in energy to the occupied $2 s$ one. ${ }^{11}$ For this reason, and having two valence electrons, beryllium is able to form two collinear molecular bonds through the formation of two hybrid $s p$ orbitals. This is the case, for instance, of the well known linear species $\mathrm{BeH}_{2}$.

We recently performed an ab initio study on linear metal chains by varying the internuclear distance in order to investigate the transition from an insulating wave function to a metallic one. ${ }^{12,13}$ A detailed discussion of the results of this study in the case of $\mathrm{Be}_{N}$ will be reported elsewhere. In this work, we focus our attention on the formation, close to the equilibrium interatomic distance, of two partially occupied edge orbitals. This fact implies the presence of two quasidegenerate low-lying states, a singlet and a triplet. In a preliminary investigation, ${ }^{14}$ this behavior was found in the case of several beryllium chains studied at different levels of theory. In particular, multireference CI (MRCI) and multireference perturbation theory methods are needed in order to study long chains with appropriate basis sets. However, because of the smallness of the interaction energy, it is difficult to obtain a well balanced treatment for different values of the interatomic distance. For this reason, FCI results are very useful, since they can be used as a benchmark for approximated methods. Indeed, in the case of "difficult" molecular systems, the (valence) FCI method gives certainly the most reliable description. Within this formalism, the wave function is expanded in the linear space of all the Slater determinants arising from any possible excitation of the electrons from (valence) occupied orbitals into virtual orbitals. The problem with this approach is that it is restricted to the treatment of 
small basis sets and a limited numbers of atoms. For instance, in the case of linear $\mathrm{Be}_{N}$, the largest case we were able to treat with our FCI algorithm ${ }^{15,16}$ was $\mathrm{Be}_{5}$ with a rather small $(3 s 1 p)$ atomic basis set. Even for such a small case, the size of the valence FCI space is almost half a billion of symmetry-adapted Slater determinants.

In this article, it will be shown that the electronic structure of an equally spaced linear $\mathrm{Be}_{N}$ chain depends in a critical way on the interatomic distance $R$. At large distances, the beryllium atoms interact through van der Waals (vdW) interactions. Close to the equilibrium distance, the beryllium atoms are bonded by Be-Be single bonds, while two singly occupied orbitals are located at the chain extremities. They are an instance of Shockley states. ${ }^{6}$ Because of the quasidegeneracy of the edge orbitals, the two unpaired electrons there located give rise to two quasidegenerate states: a singlet ${ }^{1} \Sigma_{g}^{+}$and a triplet ${ }^{3} \Sigma_{u}^{+}$. The energy split decays exponentially as a function of the number of atoms in the chain, and for $N=5$ is of the order of magnitude of a millihartree. It should be noted that this picture is true for an isolated system: for a chain adsorbed on a surface, due to the smallness of the split, singlet and triplet states would most likely mix together via spin-orbit and spin-spin interactions.

In the present work, we discuss FCI results on linear beryllium chains up to $\mathrm{Be}_{5}$, obtained, whenever possible, by using several basis sets. As atomic orbitals (AOs), an averaged natural orbital (ANO) basis set ${ }^{17}$ with different contractions was chosen, as discussed later. In a subsequent work, these results will be used to assess the quality of complete active space self-consistent-field (CAS-SCF), truncated-CI, and perturbative approaches. This will permit us to investigate the behavior of much longer chains, which are clearly beyond the possibilities of a FCI treatment. This article is organized as follows. In Sec. II, the details of computations are given, and the basis sets used for the different chains are illustrated. In Sec. III, the energy results for the different chains are presented and discussed. Section IV deals with the presence of edge orbitals, and the singlet-triplet quasidegeneracy that arises from these orbitals is discussed. In Sec. V, the problem of extrapolating short-chain results to much longer systems is addressed. Finally, in Sec. VI, some concluding remarks are drawn.

\section{COMPUTATIONAL DETAILS}

We studied equally spaced linear chains containing $N$ beryllium atoms for several values of $N$. The behavior of these systems while permitting independent bond length relaxations has also been considered in a separate work. It turned out that, at equilibrium, the different bond lengths coincide within a few percent. Therefore, for the sake of simplicity, only identical bond lengths have been considered in this work. The SCF calculations were done using the DALTON 2.0 ab initio package, ${ }^{18}$ which was also used to produce the file containing the one- and two-electron integrals computed on the AOs. The FERRARA four-index transformation ${ }^{19}$ was subsequently used to transform AO integrals to the molecular orbital (MO) basis set. The FCI calculations were performed using the BOLOGNA FCI code. ${ }^{15,16}$ The FCI pro-
TABLE I. Dimension of the basis set and FCI space, as a function of $N$, for the different basis sets.

\begin{tabular}{lccc}
\hline \hline System & Basis & $N_{\mathrm{AO}}$ & $N_{\mathrm{FCI}}$ \\
\hline $\mathrm{Be}_{2}$ & $3 s 1 p$ & 12 & 309 \\
& $4 s 2 p$ & 20 & 3393 \\
& $4 s 2 p 1 d$ & 30 & 18804 \\
& $5 s 3 p 2 d$ & 48 & 139001 \\
& $5 s 3 p 2 d 1 f$ & 62 & 399348 \\
& & & \\
$\mathrm{Be}_{3}$ & $3 s 1 p$ & 18 & 26781 \\
& $4 s 2 p$ & 30 & 1103361 \\
& $4 s 2 p 1 d$ & 45 & 16566336 \\
& $5 s 3 p 2 d$ & 72 & 344545308 \\
$\mathrm{Be}_{4}$ & $3 s 1 p$ & 24 & 2949353 \\
& $4 s 2 p$ & 40 & 436523904 \\
$\mathrm{Be}_{5}$ & $3 s 1 p$ & 30 & 353048916 \\
\hline \hline
\end{tabular}

gram is interfaced to the DALTON and the FERRARA transformation codes via the Q5Cost library. ${ }^{20-22}$

The Gaussian ANO basis sets optimized by Widmark et $a l .{ }^{17}$ were used in this work. In particular, a series of five contractions of increasing size $(3 s 1 p, 4 s 2 p, 4 s 2 p 1 d, 5 s 3 p 2 d$, and $5 s 3 p 2 d 1 f$ ) was selected. Because of the size of the FCI spaces, we were able to use all these basis sets in the case of $\mathrm{Be}_{2}$ only. Smaller basis sets were used on the longer chains, and in the case of $\mathrm{Be}_{5}$ the only basis that could be used was the $3 s 1 p$ one.

Equally spaced linear chains have a $D_{\infty h}$ symmetry. However, due to the restriction to use Abelian symmetry groups in the FCI code, all the calculations were performed in the subgroup $D_{2 h}$. In Table I, the size of the basis sets and the FCI spaces (in $D_{2 h}$ ) are reported for the different chains and basis sets considered in the study. We notice that, in the FCI calculations, all the $1 s$ orbitals were kept frozen at the SCF level and doubly occupied. This means that the $N$ lowest-energy orbitals are omitted from the FCI orbitals for each calculation. Our largest calculations, $\mathrm{Be}_{5}$ with the $3 s 1 p$ basis set and $\mathrm{Be}_{4}$ with $4 s 2 p$, are associated with FCI spaces of almost half a billion of symmetry-adapted Slater determinants. For this reason, only a limited set of distances was considered in these cases.

\section{FCI RESULTS FOR BERYLLIUM CHAINS}

The results obtained on small beryllium chains $(N=2,3$, 4, and 5) are presented in this section. We discuss the chain energies as a function of the basis set, and different values of the interatomic distance between nearest neighbors $R$ and number of Be atoms $N$. In all cases, the ground state is a totally symmetric singlet state ${ }^{1} \Sigma_{g}^{+}$. It turns out that the first excited state is an antisymmetric triplet state ${ }^{3} \Sigma_{u}^{+}$. It becomes quasidegenerate with the ground state for distances close to the energy minimum in the case of the largest chains. Other low-lying excited states are worth to be considered. In particular, two charge-transfer states, the ionic ${ }^{1} \Sigma_{u}^{+}$state and the second ${ }^{1} \Sigma_{g}^{+}$state, can be obtained with different fillings of the two edge orbitals. These states are likely to play a special 


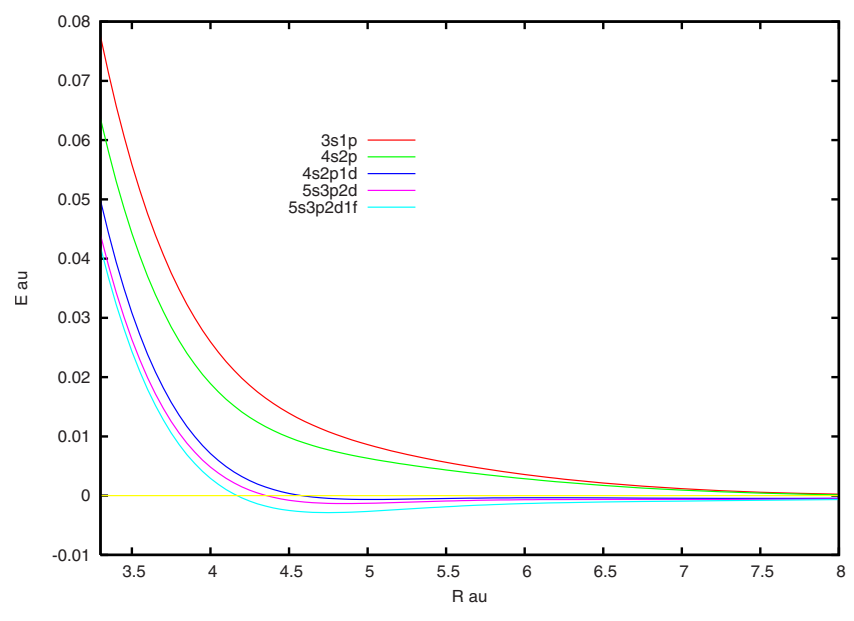

FIG. 1. (Color online) $\mathrm{Be}_{2}$ singlet total energies, with respect to the corresponding dissociation values, obtained with the $3 s 1 p, 4 s 2 p, 4 s 2 p 1 d$, $5 s 3 p 2 d$, and $5 s 3 p 2 d 1 f$ basis sets. Distances are in bohr and energies are in hartree.

role, however, only in the presence of a longitudinal electric field, which could in some case even affect the energy ordering of the states. (Notice that a longitudinal field lowers the system symmetry group to $C_{\infty v}$, and the states are no longer associated to $g$ and $u$ symmetries.) The behavior of the system in the presence of an electric field is beyond the scope of this investigation, and will be the subject of future works. In this article, we focus our attention on the singlet ground state ${ }^{1} \Sigma_{g}^{+}$and the first triplet excited state ${ }^{3} \Sigma_{u}^{+}$only.

In the case of $\mathrm{Be}_{2}$, a fairly complete basis set study is possible. In Fig. 1, we report the energy curves obtained with the $3 s 1 p, 4 s 2 p, 4 s 2 p 1 d, 5 s 3 p 2 d$, and $5 s 3 p 2 d 1 f$ basis. It appears that the energy profile is extremely sensitive to the quality of the basis set used for the calculation. This is a well known characteristic of this weakly bonded species. ${ }^{23-25} \mathrm{By}$ using the smallest basis sets $3 s 1 p$ and $4 s 2 p$, the energy curve is essentially repulsive (except for an extremely flat vdW minimum at large distance). It is the introduction of the first $d$ set of orbitals that dramatically changes the energy curve, giving rise to a shallow minimum around $R=5$ bohr. The depth of the minimum is further increased by using the two

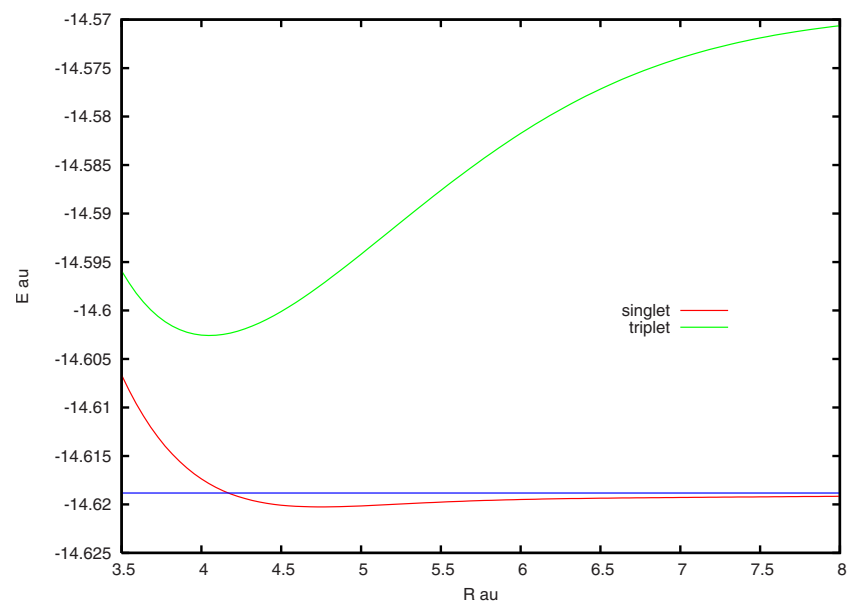

FIG. 2. (Color online) $\mathrm{Be}_{2}$ singlet and triplet energies (per Be atom) obtained with the $5 s 3 p 2 d 1 f$ basis set. Distances are in bohr and energies are in hartree.

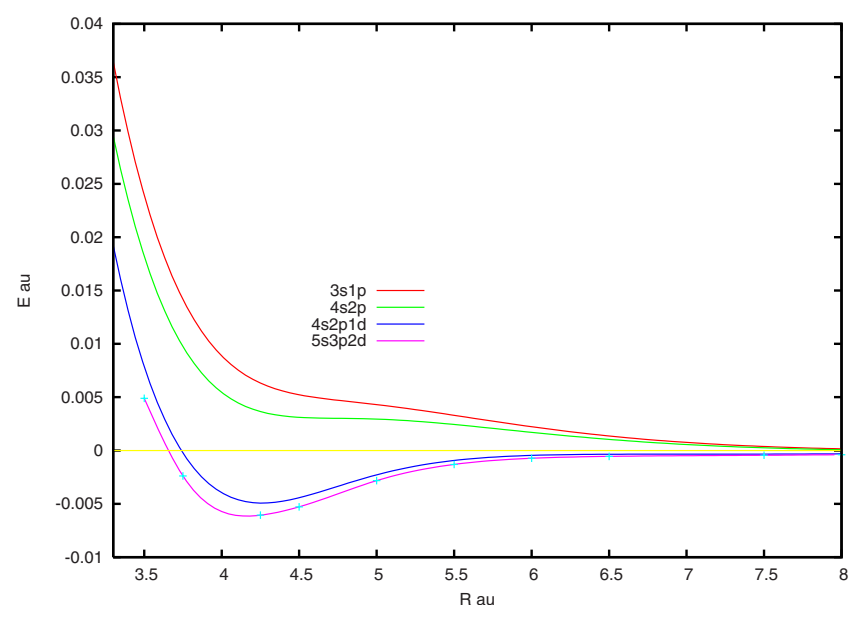

FIG. 3. (Color online) $\mathrm{Be}_{3}$ singlet total energies, with respect to the corresponding dissociation values, obtained with the $3 s 1 p, 4 s 2 p$, and $4 s 2 p 1 d$ basis sets. Distances are in bohr and energies are in hartree.

largest basis sets, although the curve modification is only tiny in these cases. In Fig. 2, the best energy curves of the lowest singlet and triplet states of $\mathrm{Be}_{2}$ are reported. They have been obtained by using the largest basis set $5 s 3 p 2 d 1 f$. Both species are bonded, with respect to the corresponding dissociation limit, the triplet in a substantially strongest way. The minima of the two species, estimated from the potentialenergy curves by using an exponential spline interpolation technique, occur at about $R=4.75$ bohr for the singlet and $R=4.00$ bohr for the triplet. The energy split in the region of the minima is relatively large, being close to 0.02 hartree.

Similar studies have been performed and are presented for $\mathrm{Be}_{3}$ (Figs. 3 and 4), Be (Figs. 5 and 6), and Be $_{5}$ (Fig. 7). Although only the smallest basis sets can be used for the longest chains, the effect of the basis appears to be extremely important regardless of the value of $N$. In particular, the introduction of the first $d$ orbital has a dramatic effect on $\mathrm{Be}_{2}$ and $\mathrm{Be}_{3}$. The singlet minimum becomes deeper and deeper as $N$ is increased, the equilibrium distance converging to a value close to $R=4.25$ for large values of $N$. A barrier to dissociation is present, close to the distance of $R=5 \mathrm{bohr}$ if

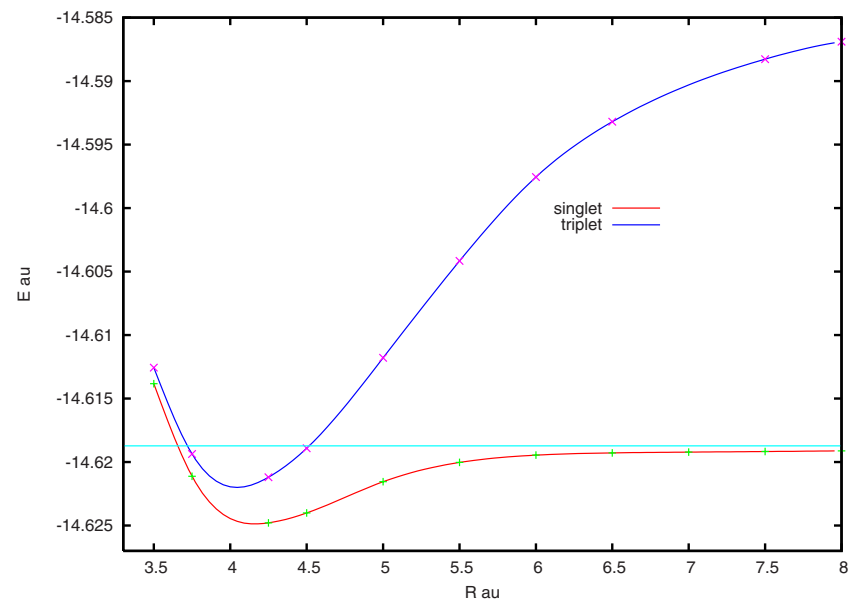

FIG. 4. (Color online) $\mathrm{Be}_{3}$ singlet and triplet energies (per Be atom) obtained with the $5 s 3 p 2 d$ basis set. Distances are in bohr and energies are in hartree. 


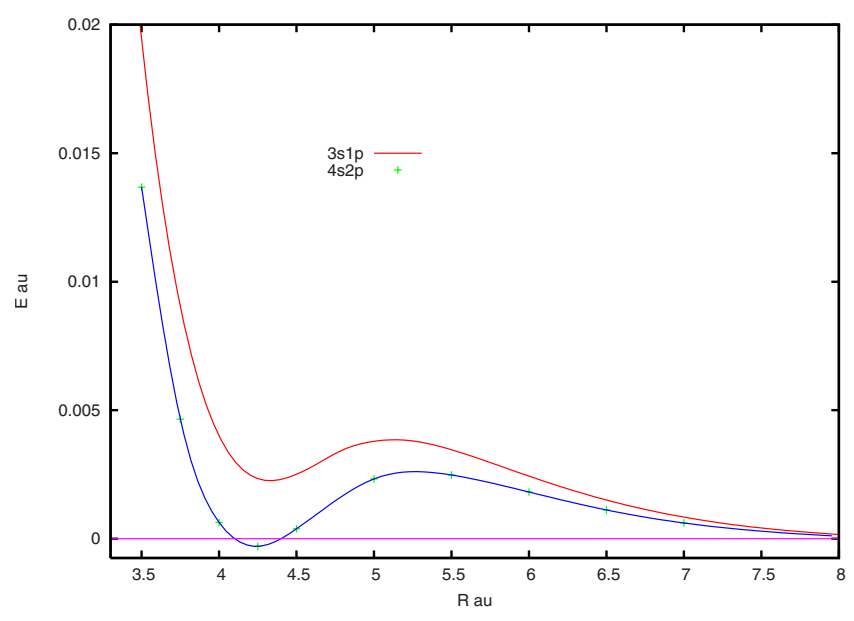

FIG. 5. (Color online) $\mathrm{Be}_{4}$ singlet total energies, with respect to the corresponding dissociation values, obtained with the $3 s 1 p$ and $4 s 2 p$ basis sets. Distances are in bohr and energies are in hartree.

the smallest basis sets are used. In the case of large basis sets, this barrier disappears. Therefore, because of the dependence on the basis set size, this feature appears to be merely artifactual. For longer chains, the triplet energy profile becomes closer and closer to the singlet one in the region of the minimum, while the energy curves are well split apart for relatively large values of $R$. It appears from these pictures that the short-distance singlet and triplet states quickly become quasidegenerate for large values of $N$, a fact that will be discussed in detail in Sec. IV.

In Table II we report, for each chain and basis set, the interpolated equilibrium energies $E_{\mathrm{S}}$ and $E_{\mathrm{T}}$, the corresponding equilibrium distances $R_{\mathrm{S}}$ and $R_{\mathrm{T}}$, the singlet dissociation (or fragmentation) energy $D_{\mathrm{S}}$, and the singlet-triplet energy split $\Delta_{\mathrm{ST}}$. As previously stated the parameters have been interpolated by using an exponential spline technique. The crucial role played by $d$ orbitals for $\mathrm{Be}_{2}$ and $\mathrm{Be}_{3}$ is confirmed: With the $3 s 1 p$ and $4 s 2 p$ basis sets, these species show only a tiny vdW minimum at long distance. The singlet-triplet energy split at equilibrium becomes very small for the largest values of $N$. It is strongly dependent on the basis-set quality,

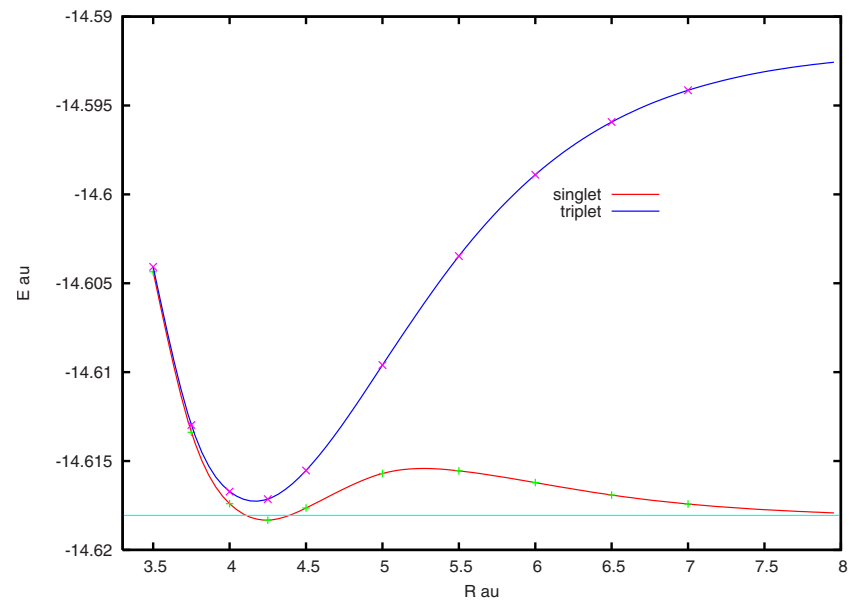

FIG. 6. (Color online) $\mathrm{Be}_{4}$ singlet and triplet energies (per Be atom) obtained with the $4 s 2 p$ basis set. Distances are in bohr and energies are in hartree.

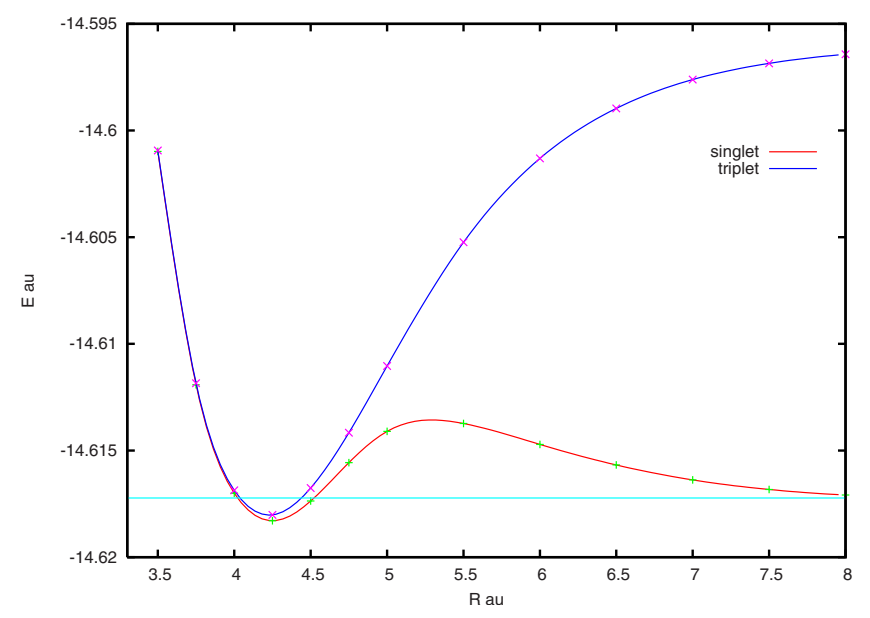

FIG. 7. (Color online) $\mathrm{Be}_{5}$ singlet and triplet energies (per Be atom) obtained with the $3 s 1 p$ basis set. Distances are in bohr and energies are in hartree.

the largest basis sets giving the smallest split values. The triplet equilibrium distance is systematically shorter than the singlet one. This is probably due to the fact that the dissociation limits of the triplets are much higher in energy than the corresponding limits of the singlets, as discussed later. However, as far as the singlet and triplet states become degenerate in the equilibrium region, $R_{\mathrm{S}}$ and $R_{\mathrm{T}}$ tend to coincide. The singlet fragmentation energy is very small for $\mathrm{Be}_{2}$ and becomes progressively larger for the longest chains. Moreover, this quantity is extremely sensitive to the basis set used and is strongly increased when the largest basis sets are employed.

Because of the crucial dependence of the (singlet) energy well minimum on the presence of $d$ functions in the expansion basis set, we also compared our FCI values for the $\mathrm{Be}_{5}$ chain with the MRCI results obtained with the $5 s 3 p 2 d 1 f$ basis set. ${ }^{12}$ In particular in this case a minimum for the singlet is found at a Be-Be distance of 4.189 bohr. The chain is characterized by a fragmentation energy of 0.0327 hartree. The singlet-triplet splitting results in $2.91 \times 10^{-4}$ hartree, while the triplet equilibrium distance is 4.186 bohr. These results globally confirm the shape of the curve on the minimum region as well as the existence of the singlet-triplet quasidegeneracy. Anyway, it has to be underlined that approximated (truncated-CI) methods tend systematically to overestimate the fragmentation energy due to the difficulty to correctly treat the dissociation region. A more systematic study of the behavior of different approximated methods will be the subject of a forthcoming paper.

As far as the long-distance behavior of the triplet is concerned, one may guess that this state dissociates to a manifold of $N$ degenerate states. These triplets are symmetry combinations of a triplet on one beryllium atom with $N-1$ singlets on the other atoms. Therefore their energy is given by $E_{\mathrm{T}}(1)+(N-1) E_{\mathrm{S}}(1)$, where $E_{\mathrm{T}}(1)$ and $E_{\mathrm{S}}(1)$ are the energies of an isolated beryllium atom in the triplet and singlet states, respectively. This fact has been confirmed by the computation of the excited state for the triplet state at the dissociation limit in the case of $\mathrm{Be}_{4}$. At the equilibrium, on the contrary, the first triplet excited state is much higher in en- 
TABLE II. Interpolated values from the $\mathrm{Be}_{N}$ curves. $E_{\mathrm{S}}$ and $E_{\mathrm{T}}$ are the singlet and triplet minimum total energies, respectively, while $R_{\mathrm{S}}$ and $R_{\mathrm{T}}$ are the corresponding equilibrium distances; $D_{\mathrm{S}}$ is the singlet fragmentation energy; $\Delta_{\mathrm{ST}}$ is the singlet-triplet adiabatic energy gap. If multiple minima are present, the reported values refer to the one corresponding to the shortest bond length. Distances are in bohr, and energies are in hartree.

\begin{tabular}{|c|c|c|c|c|c|c|}
\hline Basis & $E_{\mathrm{S}}$ & $E_{\mathrm{T}}$ & $R_{\mathrm{S}}$ & $R_{\mathrm{T}}$ & $D_{\mathrm{S}}$ & $\Delta_{\mathrm{ST}}$ \\
\hline \multicolumn{7}{|c|}{$\mathrm{Be}_{2}$} \\
\hline $3 s 1 p$ & -29.234603 & -29.173684 & 9.971 & 4.247 & 0.000066 & 0.060919 \\
\hline $4 s 2 p$ & -29.236170 & -29.185021 & 9.696 & 4.289 & 0.000108 & 0.051149 \\
\hline $4 s 2 p 1 d$ & -29.237462 & -29.198584 & 5.025 & 4.103 & 0.000643 & 0.038878 \\
\hline $5 s 3 p 2 d$ & -29.238795 & -29.202321 & 4.858 & 4.044 & 0.001334 & 0.036474 \\
\hline $5 s 3 p 2 d 1 f$ & -29.240510 & -29.205166 & 4.746 & 4.045 & 0.002849 & 0.035334 \\
\hline \multicolumn{7}{|c|}{$\mathrm{Be}_{3}$} \\
\hline $3 s 1 p$ & -43.8519415 & -43.8177281 & 9.955 & 4.243 & 0.000134 & 0.034213 \\
\hline $4 s 2 p$ & -43.8543220 & -43.8285171 & 9.630 & 4.159 & 0.000225 & 0.025577 \\
\hline $4 s 2 p 1 d$ & -43.8700101 & -43.8599106 & 4.279 & 4.124 & 0.014781 & 0.010100 \\
\hline $5 s 3 p 2 d$ & -43.8745069 & -43.8654797 & 4.220 & 4.141 & 0.018322 & 0.009027 \\
\hline \multicolumn{7}{|c|}{$\mathrm{Be}_{4}$} \\
\hline $3 s 1 p$ & -58.459979 & -58.455014 & 4.368 & 4.238 & 0.009098 & 0.004965 \\
\hline $4 s 2 p$ & -58.473315 & -58.469372 & 4.243 & 4.187 & 0.001196 & 0.003943 \\
\hline \multicolumn{7}{|c|}{$\mathrm{Be}_{5}$} \\
\hline $3 s 1 p$ & -73.091468 & -73.09013 & 4.250 & 4.233 & 0.005127 & 0.001338 \\
\hline
\end{tabular}

ergy. As an example, in the case of $\mathrm{Be}_{4}$, the $\mathrm{FCI}$ energy difference between the lowest and the first excited triplet is about 0.08 hartree (with the $3 s 1 p$ basis set).

FCI energies are often used as benchmark values for approximated methods. For this reason, we report in this work FCI energy values at a distance close to the minimum and at the dissociation. In Table III, FCI total energies are reported, for all the chains and the basis sets used, at a distance of $R=4.25$ bohr (singlet and triplet), and for $R=\infty$ (singlet only). These values will also be used in order to extrapolate to infinite systems the singlet-triplet split and the fragmentation energy of the chain (see Sec. V).

TABLE III. Singlet and triplet $\mathrm{Be}_{N}$ total FCI energies (hartree) close to the equilibrium distance $(R=4.25 \mathrm{bohr})$ and at infinite distance (for the singlet only).

\begin{tabular}{lcccc}
\hline \hline System & Basis & ${ }^{1} \Sigma_{g}^{+}$ & ${ }^{3} \Sigma_{u}^{+}$ & Infinite distance \\
\hline $\mathrm{Be}_{2}$ & $3 s 1 p$ & -29.21593209 & -29.17368330 & -29.23453766 \\
& $4 s 2 p$ & -29.22284991 & -29.18115090 & -29.23606202 \\
& $4 s 2 p 1 d$ & -29.23428495 & -29.19797121 & -29.23681864 \\
& $5 s 3 p 2 d$ & -29.23653942 & -29.20113105 & -29.23746019 \\
& $5 s 3 p 2 d 1 f$ & -29.23857674 & -29.20402840 & -29.23765039 \\
$\mathrm{Be}_{3}$ & $3 s 1 p$ & -43.83284190 & -43.81772551 & -43.85180720 \\
& $4 s 2 p$ & -43.84314271 & -43.82810769 & -43.85409429 \\
& $4 s 2 p 1 d$ & -43.87001754 & -43.85883920 & -43.85522978 \\
& $5 s 3 p 2 d$ & -43.87436964 & -43.86367574 & -43.85618466 \\
$\mathrm{Be}_{4}$ & $3 s 1 p$ & -58.45972304 & -58.45499602 & -58.46907675 \\
& $4 s 2 p$ & -58.47331060 & -58.46854629 & -58.47211917 \\
$\mathrm{Be}_{5}$ & $3 s 1 p$ & -73.09146762 & -73.09008951 & -73.08634071 \\
\hline \hline
\end{tabular}

\section{EDGE ORBITALS AND SINGLET-TRIPLET DEGENERACY}

The FCI results illustrated in Sec. III can be summarized as follows.

(1) Beryllium equally spaced linear chains have a minimum energy at an internuclear distance of about 4.25 bohr.

(2) The depth of the per-atom energy well increases as a function of the number of atoms in the chain. It increases as a function of the quality of the basis set too, the presence of at least one set of $d$ orbitals being crucial in order to achieve a qualitatively correct description of the bonds.

(3) The first excited state is a triplet. The singlet-triplet energy split is small, and it quickly shrinks to zero for large values of $N$.

These results can be rationalized by considering the nature and structure of the $\mathrm{Be}-\mathrm{Be}$ single bonds in these chains. The electronic structure of beryllium chains depends in a critical way on the interatomic distance $R$. At large distances, the beryllium atoms are essentially isolated atoms in their ground state, and the system is weakly bonded by dispersion (or vdW) interactions. This fact is confirmed by the structure of the valence orbitals, which are essentially different combinations of the $2 s$ orbitals of the Be atoms. At a distance of about $5.5 \mathrm{bohr}$, the wave function has a rapid change toward a totally different structure. This is the result of the formation, in the direction of the chain axis (say, $z$ ), of two $s p_{z}$-hybrid orbitals on each $\mathrm{Be}$ atom. In this way, $\mathrm{Be}-\mathrm{Be}$ single bonds are formed between adjacent atoms. In a $\mathrm{Be}_{N}$ chain, these bond orbitals can globally host $2 N-2$ out of the 


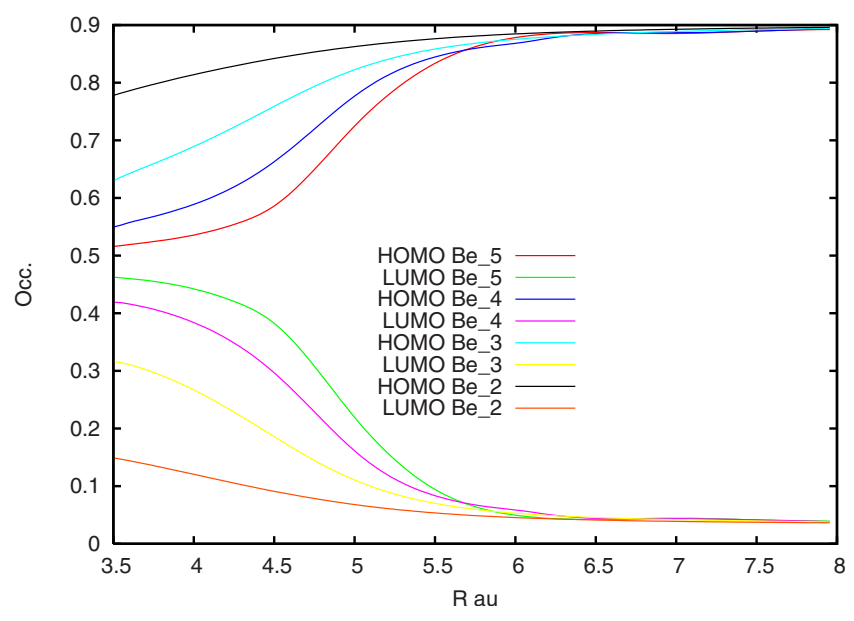

FIG. 8. (Color online) The occupation numbers of the FCI natural orbitals that correspond to the HOMO and LUMO SCF orbitals as a function of the internuclear distance $R$ and for different values of $N$.

$2 N$ valence electrons. This leaves the two terminal hybrid orbitals (edge orbitals) free at the chain ends since they cannot combine with other valence orbitals.

The edge orbitals have a higher energy than the bond orbitals that are located in the inner part of the chain. For this reason, the two remaining valence electrons are located in the edge orbitals, leaving them partially filled. Because of the quasidegeneracy of the edge orbitals (they are located at the two extremities of the molecule) the two unpaired electrons give rise to two quasidegenerate states: a singlet ${ }^{1} \Sigma_{g}^{+}$and a triplet ${ }^{3} \Sigma_{u}^{+}$. The multireference character of the singlet openshell state, as well as the evolution of the wave function nature with respect to the atom-atom distance, can be seen in Fig. 8. In this figure, the occupation of the FCI natural orbital spin orbitals (with the $3 s 1 p$ basis) is reported as a function of the Be-Be distance for different values of $N$. In particular it appears that, at a distance of about $5.5 \mathrm{bohr}$, the natural orbitals corresponding to the edge orbitals [which correspond to the highest occupied $\mathrm{MO}$ (HOMO) and lowest unoccupied MO (LUMO) of the SCF calculation] experience an important change: at this distance, their occupation numbers go from a closed-shell (long distance) to an open-shell situation. This behavior is present in all chains and becomes sharper by increasing the value of $N$. Indeed, in the case of $\mathrm{Be}_{5}$, the two edge orbitals at distances close to the equilibrium have an occupation close to 1 .

The situation is very much reminiscent of that of a dissociated hydrogen molecule, which presents a similar singlet-triplet degenerate pattern. In beryllium chains, however, the space between the two unpaired electrons is not empty but contains all the nuclei and electrons of the molecule. In particular, all the other valence electrons of the system are located in this region of space. This explains the crucial role of dynamical electron correlation in the energetics of these systems. In particular, the two extreme situations (close to the energy minimum and at infinite distance) are associated with very different correlation effects. At FCI level the differential correlation effects are described in an exact way (within the chosen basis set). On the other hand, things are completely different when approximate methods are used, as discussed in a forthcoming paper.

\section{LONG-CHAIN EXTRAPOLATION}

The per-atom binding energy shows a marked increasing behavior as a function of $N$. This kind of phenomenon is sometimes interpreted as due to the presence of cooperative effects. In the case of beryllium chains, their presence seems rather unlikely due to the strong covalent nature of the $\mathrm{Be}-\mathrm{Be}$ bond at the equilibrium distance. In this section, it will be shown how the singlet and triplet energies can be rationalized through a simple phenomenological picture, which does not require the introduction of any cooperative effect. The two ingredients of the scheme are

(1) an antiferromagnetic interaction between the two terminal unpaired electrons of the chain and

(2) a simple additive expression for the chain energy, taking into account the number of $\mathrm{Be}-\mathrm{Be}$ bonds and the presence of the terminal radical $\mathrm{Be}$.

As will be discussed in a forthcoming paper, this phenomenological scheme can be very useful in order to estimate the binding energy of long chains by performing calculations on the shortest ones. In order to avoid the introduction of further degrees of freedom, we fixed the internuclear distance to the value of $R=4.25 \mathrm{bohr}$, which is very close to the equilibrium distance. We denote by $E_{\mathrm{S}}(N)$ and $E_{\mathrm{T}}(N)$ the singlet and triplet energies of the $\mathrm{Be}_{N}$ chain computed for this value of the distance. The values of $E_{\mathrm{S}}(N)$ and $E_{\mathrm{T}}(N)$, obtained with the different basis sets, are collected in Table III.

It is possible to interpret these energies as due to a Heisenberg interaction of antiferromagnetic type $J(N)$ between the two terminal unpaired electrons. In this schema, the energy pattern is the result of a split around a "mean energy" value $E_{\mathrm{ST}}(N)$ due to the presence of the coupling $J(N)$,

$$
E_{\mathrm{S}}(N)=E_{\mathrm{ST}}(N)-J(N)
$$

and

$$
E_{\mathrm{T}}(N)=E_{\mathrm{ST}}(N)+J(N) .
$$

For the distance dependence of the coupling $J(N)$, a simple exponentially decreasing behavior of the type

$$
J(N)=J_{0} \exp (-N / \nu)
$$

is often assumed. In Fig. 9 (zoom in Fig. 10), the logarithm of $J(N)$ is plotted against $N$. Notice that, since $R$ is fixed, $N$ is proportional to the chain length, and hence roughly proportional to the distance between the two unpaired electrons. The linear behavior of the logarithm clearly indicates that $J(N)$ has an exponential decay as a function of the distance. In the case of the $3 s 1 p$ basis set, where more points have been computed, we also performed a least squares fitting of the linearized expression for $J$. The interpolated parameters are $J_{0}=0.219438$ hartree, while $1 / \nu=-1.1431$. The correlation coefficient is equal to -0.9992 , thus confirming a satisfactory linear behavior. The coupling is weakly dependent on 


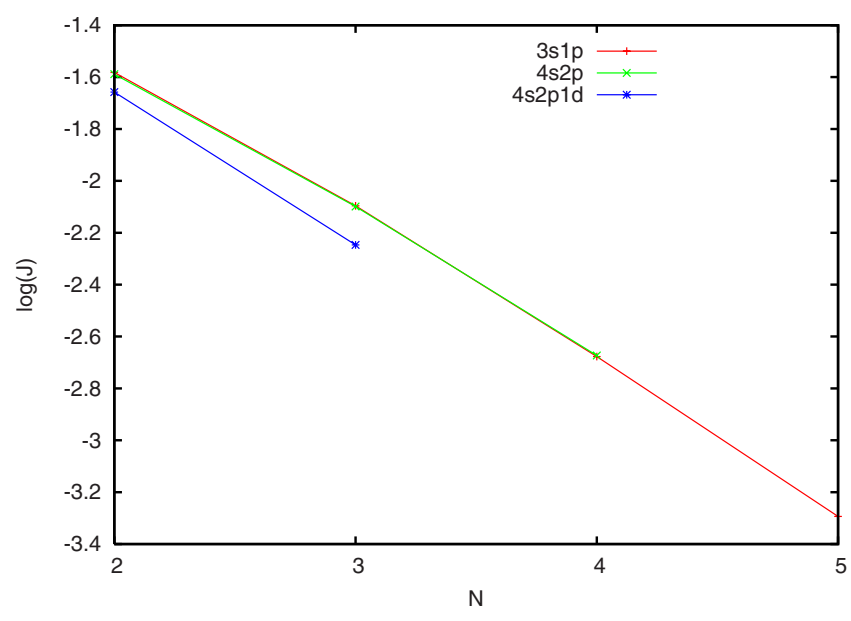

FIG. 9. (Color online) Singlet-triplet energy split (logarithmic scale) as a function of $N$ for the different $3 s 1 p, 4 s 2 p$, and $4 s 2 p 1 d$ basis sets. $R$ is fixed at 4.25 bohr; energy split is in hartree.

the basis set: a very moderate increase in $J$ as the basis set is enlarged can be observed. The main effect appears to be due to the addition of the first set of $d$ orbitals, a fact that is recurrent for this type of systems (see the discussion on the fragmentation energy later in this section). In any case, by passing from the $3 s 1 p$ basis set to the $5 s 3 p 2 d 1 f$ one, the value of $J$ increases by about $6 \%$ only.

We come now to the problem of the binding energy. The presence of "cooperative effects" has often been evoked in order to explain the strong dependence of the average (i.e., per atom) potential-energy curve on the number of atoms in the chain. However, it should be noticed that open chains can present important edge effects that are able to induce a similar behavior. In order to elucidate this point, a linear fit of the chain energy was performed by using the following functional form for the $E_{\mathrm{ST}}(N)$ average energy:

$$
E_{\mathrm{ST}}(N)=(N-1) E_{\mathrm{Be}-\mathrm{Be}}+E_{\cdot \mathrm{Be}} \cdot
$$

Such an expression takes into account a purely local Be-Be energy contribution $\left(E_{\mathrm{Be}-\mathrm{Be}}\right)$ proportional to the number of bonds, and a fixed contribution due to the two terminal atoms (indicated conventionally by $E_{\text {. }}$.). The term $E_{\mathrm{Be}-\mathrm{Be}}$ is to be multiplied by $N-1$ (the number of Be-Be bonds), while $E_{\text {. Be }}$. describes the singlet-triplet averaged effect of the two terminal electrons. Notice that this is the simplest expression that can be used for a finite linear chain, and it clearly does not include any cooperative effect.

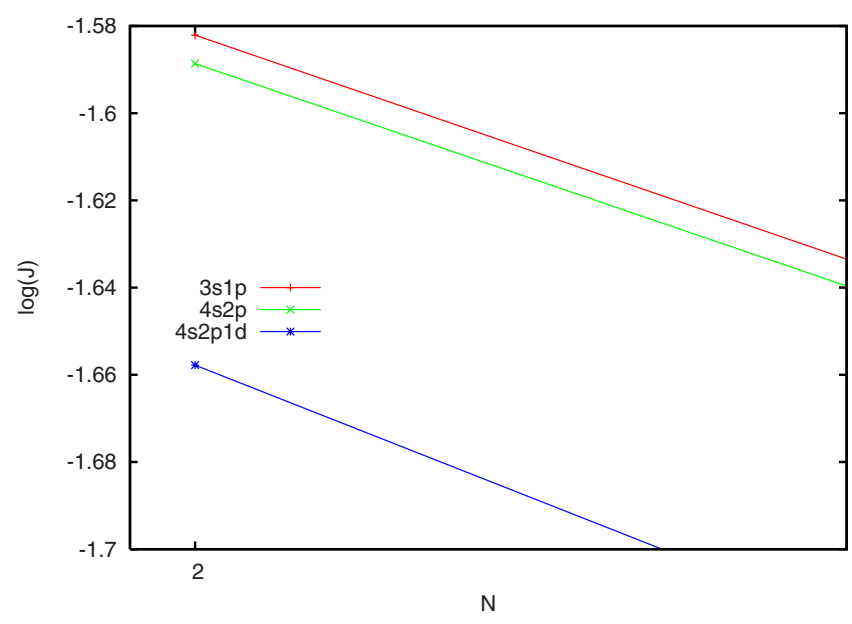

FIG. 10. (Color online) A zoom of the previous figure.

In Table IV, fitted energies obtained by using four different basis sets are reported. For the sake of comparison, the isolated-atom energy $E_{\mathrm{Be}}$ obtained at the same level of calculation is also reported. With the smallest basis set, it was possible to use two different sets of values of $N$ to extract the infinite-chain parameters. In this case we also performed a least square fitting using the $E_{\mathrm{ST}}$ values computed for $N$ $=2,3,4,5$. By comparing the values obtained with $N=2,3$, $N=4,5$, and the least squares fitting with the complete set, one can see a remarkable stability of $E_{\mathrm{Be}-\mathrm{Be}}$ and $E_{\text {.Be., }}$, regardless of the values of $N$ used to extract the parameters. The least squares fitting shows a really high correlation, the correlation coefficient $\rho$ being equal to -0.999999998 . Moreover the deviation from computed and (least square) interpolated values of $E_{\mathrm{ST}}$ is really negligible, being of the order of $7 \times 10^{-4}$ hartree for all the points. These facts clearly indicate the absence of any important cooperative effect in the chain energy. Figure 11 shows the fitted energies as a function of $N$ (considered as a real parameter), obtained with the smallest basis set $3 s 1 p$. In the same figure we also report the values of the computed $E_{\mathrm{ST}}$ and the values of the singlet and triplet energies obtained by the interpolated values of $E_{\mathrm{ST}}$ and the least square fitted values of $J$, following Eqs. (1) and (2). The high agreement is again a confirmation of the validity of our interpolation scheme as well as of the exponential behavior of $J$. By using the fitted value of the bond energy $E_{\mathrm{Be}-\mathrm{Be}}$ and the atomic energy $E_{\mathrm{Be}}$, it is possible to estimate the fragmentation energy (per atom) of the infinite chain. The

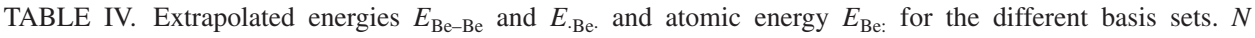
indicates the values used for the extrapolation, while $D_{\infty}$ denotes the estimated fragmentation energy for the infinite chain. All energies are in hartree.

\begin{tabular}{lccccc}
\hline \hline Basis & $N$ values & $E_{\mathrm{Be}-\mathrm{Be}}$ & $E_{\text {.Be }}$ & $E_{\mathrm{Be}:}$ & $D_{\infty}$ \\
\hline $3 s 1 p$ & 2,3 & -14.63047601 & -14.56433169 & -14.61726883 & 0.01320718 \\
& 4,5 & -14.63341903 & -14.55710243 & -14.61726883 & 0.01615020 \\
& Least squares $^{\mathrm{a}}$ & -14.63199884 & -14.56206027 & -14.61726883 & 0.01473001 \\
$4 s 2 p$ & 2,3 & -14.63362480 & -14.56837561 & -14.61803101 & 0.01559379 \\
$4 s 2 p 1 d$ & 2,3 & -14.64830029 & -14.56782779 & -14.61840932 & 0.02989097 \\
$5 s 3 p 2 d$ & 2,3 & -14.65018746 & -14.56864778 & -14.61873010 & 0.03145736 \\
\hline \hline
\end{tabular}

abtained from a least squares fitting in the interval $N=(2,5)$. Correlation coefficient $\rho=0.999999998$. 


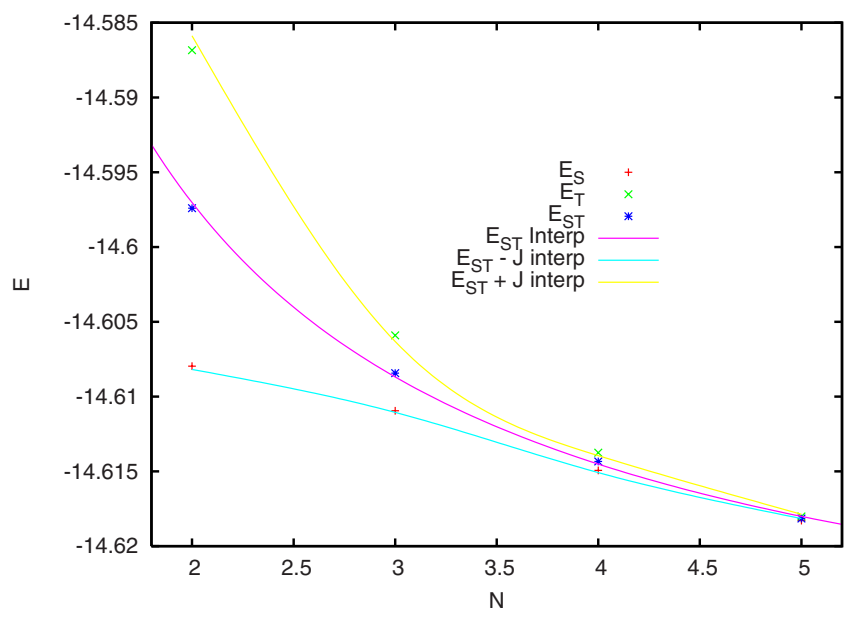

FIG. 11. (Color online) Extrapolated mean energies $E_{\mathrm{ST}}$ as a function of $N$ and with the $3 s 1 p$ basis set. $R$ is fixed at 4.25 bohr; energies are in hartree.

fragmentation energy is given by $D_{\infty}=E_{\mathrm{Be}:}-E_{\mathrm{Be}-\mathrm{Be}}$. The absence of cooperative effects means that it is possible to use small clusters in order to get a reliable estimation of the fragmentation energy of the infinite chain. This is a remarkable result, especially if one considers the fact that the fragmentation energy of short chains has a completely different value from the corresponding infinite-system limit.

The fragmentation energy turns out to be extremely sensitive to the basis set used to compute it. From Table IV, it appears that $D_{\infty}$ strongly increases by passing from the smallest basis sets to the largest ones. In particular, the presence of at least one set of $d$ orbitals appears to be crucial to stabilize the result. This fact agrees with the behavior already observed in the case of $\mathrm{Be}_{2}$ and seems to indicate the presence of an important dynamical correlation close to equilibrium distances of these systems. The value for $D_{\infty}$ obtained with the $3 s 1 p$ basis set is less than one-half than the corresponding value with the $5 s 3 p 2 d$ orbitals.

\section{CONCLUSIONS}

We presented a FCI study of $\mathrm{Be}_{N}(N=2-5)$ open chains. Beryllium equally spaced linear chains have a minimum energy at an internuclear distance of about 4.25 bohr. The minimum is due to the formation, close to the equilibrium distance, of $N-1 \mathrm{Be}-\mathrm{Be}$ single bonds. These bonds can be understood as coming from $s p$-hybrid AOs. The depth of the per-atom energy well increases as a function of the number of atoms in the chain. This fact, however, is not due to cooperative effects but comes from the ratio $(N-1) / N$ between the number of bonds and atoms in a chain. The energy well increases also as a function of the quality of the basis set, the presence of at least one set of $d$ orbitals being crucial in order to achieve a qualitatively correct description of the bonds.

Two dangling $s p$ orbitals are left, so beryllium linear chains present two quasidegenerate edge orbitals located at the chain extremities. This represents a nice illustration of the surface states predicted by Tamm and Shockley long ago. Although beryllium is likely to have a limited technological impact, a similar behavior is expected for magnesium, cal- cium, and the other elements of group 2. Moreover, preliminary calculations on mercury indicate that these features could be shared also by metals of group 12 . Therefore, the properties described in this work can be expected from a rather large group of metals. Investigations are on the way in order to determine the equilibrium geometries of these systems. The presence of these edge orbitals has several interesting consequences.

- The two quasidegenerate orbitals, one above and one below the Fermi level, globally host two electrons. This produces one singlet quasidegenerate state and one triplet quasidegenerate state, whose splitting quickly decreases when the chain length increases.

- The existence of ionic low-lying states is also expected. Therefore, the properties of the system in the presence of magnetic or electric fields are worth to be investigated as well as the effect of the spin-orbit coupling on these states.

- Positive ions can be obtained by extracting one electron from the system. Because of geometry distortion, such a hole will tend to localize in one of the edge orbitals. For this reason, sufficiently long chains can lower the system symmetry and give rise to bistable systems.

These aspects will be considered in detail in future works. We plan also to perform a systematic investigation concerning the other metals of groups 2 and 12.

From a methodological point of view, these systems represent a very stringent test for different approximated methods (such as CI, coupled cluster, perturbation theory, etc.) because of the difficulty of computing accurate values for both the singlet-triplet splitting and the fragmentation energy. In fact, the singlet-triplet split is extremely small, and different approximated methods give totally different values. The fragmentation energy, on the other hand, requires a well balanced description of the two different geometries, i.e., both at short and long distances. Although a CAS-SCF description is in principle able to give a correct description of these systems, it is extremely difficult to obtain a consistent set of orbitals at the different distances.

Experimentally, edifices showing this structure could perhaps be realized by deposing beryllium atoms on an inert surface. A further possibility could be to host such atomic chains within a hollow structure, such as, for instance, a nanotube. In this case, the steric constraint of the tube would prevent the collapse of the chain into compact more stable structures. Work is in progress to investigate the effects of the hosting species on the electronic structure of the chains.

\section{ACKNOWLEDGMENTS}

This work was partly supported by the French "Centre National de la Recherche Scientifique" (CNRS) and the PICS 4263 (CNRS), the Italian Ministry of University and Research (MIUR), and the University of Bologna, under the project "PRIN 2006. Molecular Quantum Mechanics: Computational Methods and Analysis of Novel Phenomena." 
Support from the European Community under the COST D37 (GRIDCHEM) action is also gratefully acknowledged.

${ }^{1}$ E. G. Emberly and G. Kirczenow, Phys. Rev. B 60, 6028 (1999).

${ }^{2}$ S. R. Bahn and K. W. Jacobsen, Phys. Rev. Lett. 87, 266101 (2001).

${ }^{3}$ F. J. Himpsel, K. N. Altmann, R. Bennewitz, J. N. Crain, A. Kirakosian, J.-L. Lin, and J. L. McChesney, J. Phys.: Condens. Matter 13, 11097 (2001).

${ }^{4}$ E. P. Amorim, A. J. R. da Silva, A. Fazzio, and E. Z. da Silva, Nanotechnology 18, 145701 (2007).

${ }^{5}$ J. N. Crain and D. T. Pierce, Science 307, 703 (2005)

${ }^{6}$ S. G. Davidson and M. Stęślicka, Basic Theory of Surface States (Clarendon, Oxford, 1992).

${ }^{7}$ O. Hod, J. E. Peralta, and G. E. Scuseria, Phys. Rev. B 76, 233401 (2007).

${ }^{8}$ O. Hod, V. Barone, and G. E. Scuseria, Phys. Rev. B 77, 035411 (2008).

${ }^{9}$ Y.-W. Son, M. L. Cohen, and S. G. Louie, Nature (London) 444, 347 (2006).

${ }^{10}$ A. Monari, G. L. Bendazzoli, and S. Evangelisti, J. Chem. Phys. 129, 134104 (2008).

${ }^{11}$ S. Salomonson, H. Warston, and I. Lindgren, Phys. Rev. Lett. 76, 3092 (1996).

${ }^{12}$ V. Vetere, A. Monari, G. L. Bendazzoli, S. Evangelisti, and B. Paulus, J. Chem. Phys. 128, 024701 (2008).
${ }^{13}$ G. L. Bendazzoli, S. Evangelisti, A. Monari, B. Paulus, and V. Vetere, J. Phys.: Conf. Ser. 117, 012005 (2008).

${ }^{14}$ A. Monari, V. Vetere, G. L. Bendazzoli, S. Evangelisti, and B. Paulus, Chem. Phys. Lett. 465, 102-105 (2008).

${ }^{15}$ G. L. Bendazzoli and S. Evangelisti, J. Chem. Phys. 98, 3141 (1993).

${ }^{16}$ L. Gagliardi, G. L. Bendazzoli, and S. Evangelisti, J. Comput. Chem. 18, 1329 (1997).

${ }^{17}$ P.-O. Widmark, P.-Å. Malmqvist, and B. O. Roos, Theor. Chim. Acta 77, 291 (1990).

${ }^{18}$ DALTON, a molecular electronic structure program, Release 2.0 (2005) (see http://www.kjemi.uio.no/software/dalton/dalton.html).

${ }^{19}$ R. Cimiraglia and C. Angeli, personal communication (April 2006).

${ }^{20}$ C. Angeli, G. L. Bendazzoli, S. Borini, R. Cimiraglia, A. Emerson, S. Evangelisti, D. Maynau, A. Monari, E. Rossi, J. Sanchez-Marin, P. G. Szalay, and A. Tajti, Int. J. Quantum Chem. 107, 2082 (2007).

${ }^{21}$ S. Borini, A. Monari, E. Rossi, A. Tajti, C. Angeli, G. L. Bendazzoli, R. Cimiraglia, A. Emerson, S. Evangelisti, D. Maynau, J. Sanchez-Marin, and P. G. Szalay, J. Chem. Inf. Model. 47, 1271 (2007).

${ }^{22}$ A. Scemama, A. Monari, C. Angeli, S. Borini, S. Evangelisti, and E. Rossi, Lect. Notes Comput. Sci. 5072, I/1094 (2008).

${ }^{23}$ B. Liu and A. D. McLean, J. Chem. Phys. 72, 3818 (1980).

${ }^{24}$ R. J. Harrison and N. C. Handy, Chem. Phys. Lett. 98, 97 (1983).

${ }^{25}$ S. Evangelisti, G. L. Bendazzoli, and L. Gagliardi, Chem. Phys. 185, 47 (1994). 